Copyright $@ 2010$ Institute of Electrical and electronics Engineers, Inc.

All Rights reserved.

Personal use of this material, including one hard copy reproduction, is permitted.

Permission to reprint, republish and/or distribute this material in whole or in part for any other purposes must be obtained from the IEEE.

For information on obtaining permission, send an e-mail message to stds-igr@ieee.org.

By choosing to view this document, you agree to all provisions of the copyright laws protecting it.

Individual documents posted on this site may carry slightly different copyright restrictions.

For specific document information, check the copyright notice at the beginning of each document. 


\title{
Impact of Multiple Clusters on Neural Classification of ROIs in Digital Mammograms
}

\author{
Brijesh Verma
}

\begin{abstract}
This paper evaluates the impact of multiple clusters on neural classification of regions of interest (ROIs) in digital mammograms. The training and test sets for neural networks usually contain inputs extracted from ROIs and relevant class such as benign and malignant. However, the patterns such as regions of interest in digital mammograms do not have just one cluster per class instead they have many clusters within benign and malignant classes. Therefore, neural network training may benefit in terms of accuracy and efficiency by creating and analyzing a number of clusters within a class. A novel multiple clusters based neural classification approach is presented. In this approach, input data is clustered into a number of clusters per class and a neural classifier is trained with clustered data which contain multiple clusters per class. The experiments on a benchmark database of digital mammograms are conducted. The results show that the multiple clusters per class have significant impact on neural classification and overall they achieve better accuracy than single cluster per class based classification of ROIs in digital mammograms.
\end{abstract}

\section{INTRODUCTION}

Breast cancer continues to be the most common cause of cancer deaths in women. Every year more than 1 million women develop breast cancer world-wide. A report by a national cancer institute estimates that 1 in 11 women develop breast cancer in Australia [1]. Breast cancer is the most common cause of cancer-related death in women in Australia. Each year more than 11,700 new cases of breast cancer and 2,600 deaths occur in Australia. In the United States an estimated 1,437,180 new cases of breast cancer occurred together with an estimated mortality of 565,550 during 2008 [2].

As there is currently no means of preventing breast cancer, the focus in reducing deaths from the disease has been on finding breast cancer as early as possible. Early detection of cancer saves patients from the more aggressive radical treatments and increases the overall survival rate. The introduction of screening mammography in 1963 brought about a major revolution in breast cancer detection and diagnosis. It is widely adopted in many countries including Australia as a nation wide public health care program. The decline in the number of breast cancer deaths corresponds directly to an increase in routine mammography screening.

Mammography is one of the best methods for early detection of breast cancer. It reduces the mortality rate by as much as $41 \%$ [3]. However various studies [4] have demonstrated that on average an estimated 21 percent of breast cancers are

Verma, B. is with CQ University, Australia (phone: +61 74930 9058; e-mail: b.verma@cqu.edu.au). missed by radiologists during screening mammography. Research showed [5-8] that Computer Aided Diagnosis (CAD) systems for breast cancer can improve the detection rate from $4.7 \%$ to $19.5 \%$ compared to radiologists. A comprehensive review of techniques and algorithms for CAD systems has been recently presented [9-11].

The brain of a CAD system is its classifier which can analyse, learn and classify ROIs/suspicious areas in digital mammograms. Neural network based learning algorithms [11-17] and other techniques [18-25] to learn from the attributes of given class patterns and to classify the unknown patterns of given classes into appropriate classes using the acquired knowledge have been used. Recent work conducted by us [13-14] has shown potential of neural networks for finding appropriate features and classifying them into benign and malignant classes.

Most of the classifiers for diagnosis of breast cancer have focused on classification of ROIs/suspicious areas in digital mammograms as a single class (benign or malignant). Almost all existing research techniques for classification of ROIs use one cluster per class which is inappropriate as abnormalities within benign and malignant class vary and can have different feature space for the same class. The use of a classifier with a single cluster per benign/malignant class has dominated the recent research in this area without achieving a consistent and satisfactory classification accuracy.

In order to improve the classification accuracy, this paper investigates the impact of multiple clusters on classification of ROIs. It presents a novel methodology that uses clustering to create multiple clusters within existing classes (benign and malignant) and incorporates multiple clusters based new classes within the neural network training process.

This paper consists of four sections. Following introduction, a research methodology is described in Section II. Section III presents the experimental results and a comparative analysis of the results. Section IV concludes the chapter.

\section{Multiple Clusters Based Neural Classifier}

A general overview of research methodology using multiple clusters based neural classifier for classification of ROIs/suspicious areas in digital mammograms is presented in Figure 1. The main aim of the research methodology in this paper is to conduct experiments with multiple clusters and find whether or not multiple clusters have any impact on classification accuracy. The research methodology used in this paper is based on two approaches as follows. The first 
approach is to create multiple clusters for each class benign and malignant and then train the neural classifier using the multiple clusters for each class. An overview of this approach is shown in Figure 2. In this approach, the digital mammograms are processed, ROIs/suspicious areas and features are extracted. A clustering algorithm is used to cluster the feature data into a number of clusters for both benign and malignant classes. After clustering, a neural classifier with input features and multiple clusters per class is trained for classification.

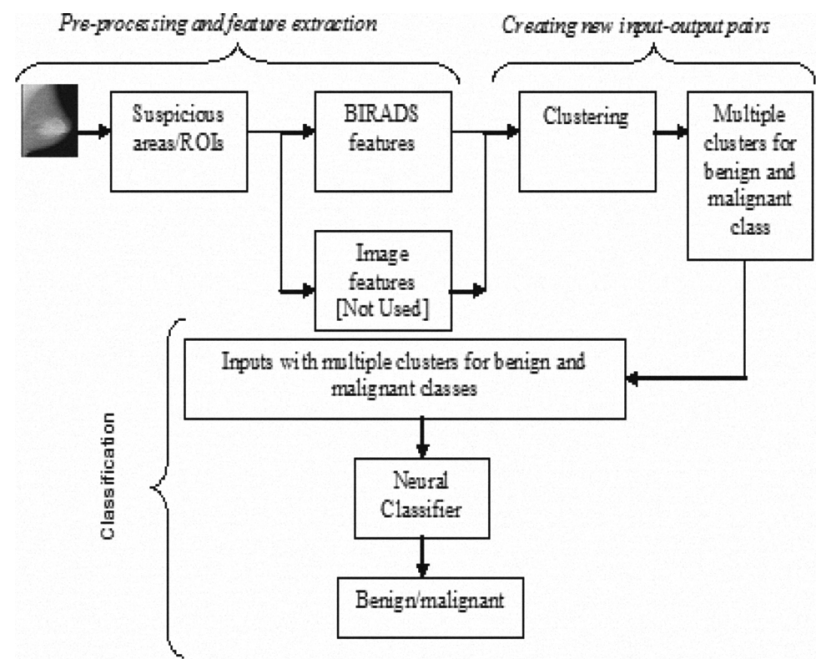

Figure 1: Overview of Research Methodology

The second approach is to use single cluster for each class which is standard approach used in neural networks. The purpose of second approach is to compare the impact of single and multiple clusters. An overview of second approach is shown in Figure 3. In this approach, the digital mammograms are processed, ROIs/suspicious areas and features are extracted same as in previous approach. Input features and outputs are used for training and testing of neural classifier.

The research methodology requires a number of steps before neural network training and testing stages. The details are given in sections below.

\section{A. Digital Mammograms and Feature Extraction}

Digital mammograms were acquired from DDSM (Digital Database for Screening Mammography) [26]. It contains approximately 2600 high quality images together with case related information. The research in this paper used 200 mammograms comprising an equal number of masses with 100 mammograms being selected for training and 100 for testing. The suspicious areas were extracted using a chain code. The chain code is provided with DDSM for each suspicious area. Six features have been extracted from suspicious areas and they represent four BI-RADS descriptor features together with patient age and a subtlety value [13]. All six features are Density, Mass Shape, Mass Margin,
Abnormality Assessment Rank, Patient Age and Subtlety Value.

\section{B. Multiple Clusters}

A clustering algorithm is used to create multiple clusters for each class. The available input patterns for benign and malignant classes are separately clustered. After clustering, new training and testing pairs are created. The number of classes for benign will be equal to the number of clusters within benign patterns. The number of classes for malignant will be equal to the number of clusters within malignant patterns.

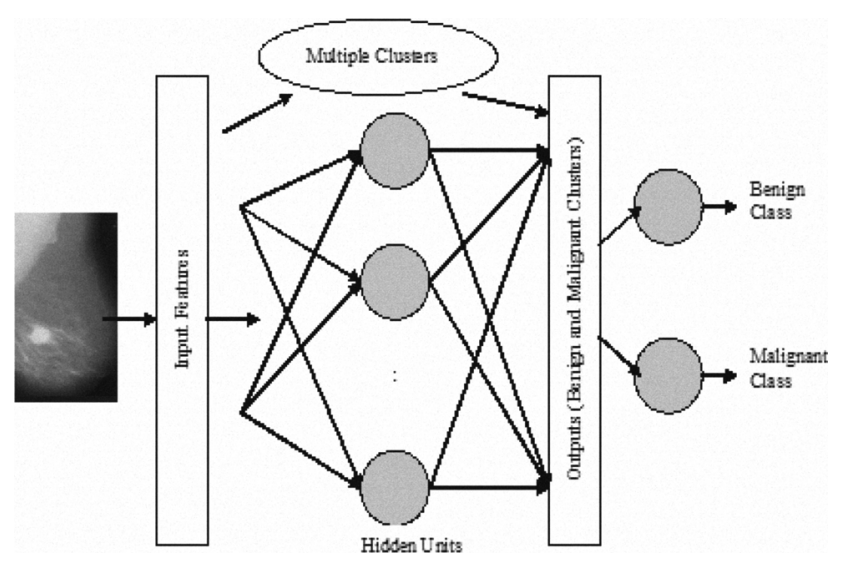

Figure 2: Multiple Clusters for Each Class (MCEC)

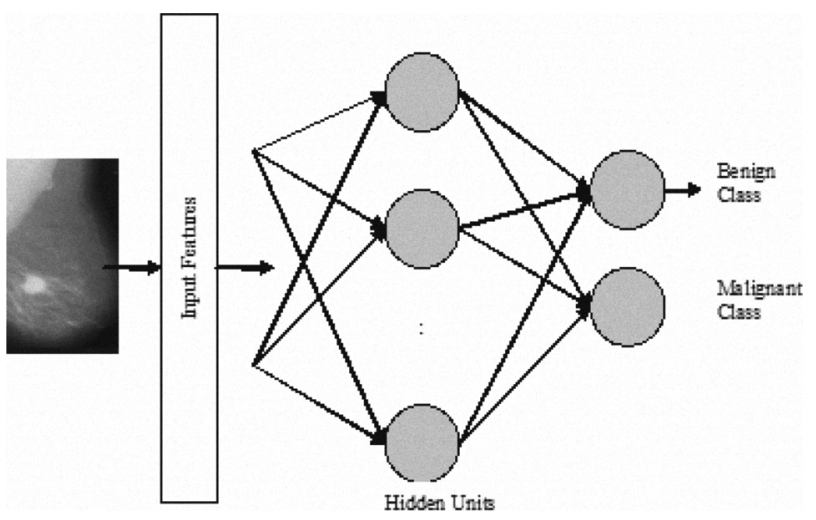

Figure 3: Single Cluster for Each Class (SCEC)

C. Neural Network Training Process

The training process for single cluster per class is standard training used in neural networks so here training of neural network using only multiple clusters is presented.

- Divide malignant and benign training data patterns

- Extract suspicious areas/regions of interest using chain code

- Extract features

- Cluster benign and malignant features into $\mathrm{k}$ clusters (where $\mathrm{k}$ is variable and it is investigated)

- Pass all training patterns to the clustering algorithm and check the number of patterns in each cluster

- Create new inputs and outputs using multiple clusters 
- Initialize weights between input and hidden units at small random values

- Calculate the output weights

D. Neural Network Testing Process

The following test process is used to test the proposed approach.

- Divide malignant and benign test data patterns

- Extract suspicious areas/regions of interest using chain code

- Extract features

- Feed input features to the network

- Check the output

- If output is one of the benign clusters, input is classified as benign otherwise malignant

The neural network classifier as shown in Figures 2 and 3 are used for classification. The neural network training algorithm used in this research is a random weights-MGS. It utilizes six input nodes to represent each input feature. The number of hidden neurons is being determined experimentally to ascertain the optimal configuration. The number of outputs is dependent on number of clusters per class.

\section{EXPERIMENTAL RESULTS AND COMPARATIVE ANALYSIS}

The multiple clusters based neural classification approach described in previous section has been evaluated on a benchmark database. Digital mammograms from the DDSM benchmark described in previous section were used for evaluation. The experiments by varying the number of hidden units and number of clusters per class have been conducted. The results are presented below in Table 1 .

TABLE 1: EXPERIMENTAL RESULTS

\begin{tabular}{|c|c|c|c|}
\hline \multirow[b]{2}{*}{ Approach } & \multirow[b]{2}{*}{$\begin{array}{l}\text { \#Hidden } \\
\text { Units }\end{array}$} & \multicolumn{2}{|c|}{ Classification Accuracy [\%] } \\
\hline & & $\begin{array}{l}\text { Training Set } \\
(50 \text { Benign } \\
\text { and } \quad 50 \\
\text { Malignant })\end{array}$ & $\begin{array}{l}\text { Test Set } \\
(50 \quad \text { Benign } \\
\text { and } 50 \\
\text { Malignant })\end{array}$ \\
\hline \multirow{2}{*}{$\begin{array}{l}\text { SCEC } \\
\mathbf{1} \text { cluster/class }\end{array}$} & 27 & 96 & 93 \\
\hline & 30 & 96 & 93 \\
\hline \multirow{2}{*}{$\begin{array}{l}\text { MCEC } \\
\mathbf{2} \text { clusters/class }\end{array}$} & 27 & 93 & 95 \\
\hline & 30 & 95 & 94 \\
\hline \multirow{2}{*}{$\begin{array}{l}\text { MCEC } \\
\mathbf{3} \text { clusters/class }\end{array}$} & 27 & 93 & 96 \\
\hline & 30 & 95 & 96 \\
\hline \multirow{2}{*}{$\begin{array}{l}\text { MCEC } \\
4 \text { clusters/class }\end{array}$} & 27 & 92 & 95 \\
\hline & 30 & 93 & 95 \\
\hline
\end{tabular}

The proposed multiple clusters for each class (MCEC) based classification approach has been compared with single cluster for each class (SCEC) based classification approach. As shown in Table 1, the results obtained by using multiple clusters are dependent on the number of clusters used in each class. The classification accuracy on test data increases with the increase in the number of clusters. The results on training data were less consistent and classification accuracy decreases as number of clusters increases. However it should be noted that multiple clusters based approach MCEC (2 clusters/class, 3 clusters/class, 4 clusters/class) was always better than the single cluster/class based approach SCEC.

\section{CONCLUSIONS AND FUTURE RESEARCH}

This paper has investigated multiple clusters based neural network to find out whether or not multiple clusters have any impact on classification of ROIs in digital mammograms. The experimental results support that the multiple clusters per class improve the classification accuracy. It was found that the accuracy increases with the increase of number of clusters per class. However, the classification accuracy starts dropping after a certain threshold.

Based on experimental results, it can be concluded that the multiple clusters per class for classification of ROIs into benign and malignant make significant impact on improving overall classification accuracy.

Future research will focus on developing an algorithm for finding the accurate number of clusters and significance of each cluster.

\section{REFERENCES}

[1] National Breast Cancer Foundation, Fast facts about breast cancer in Australia, http://www.nbcf.org.au/, accessed on 22 December 2008.

[2] American Cancer Society, Breast cancer facts and figures 2007-2008, http://www.cancer.org/ accessed on 22 December 2008.

[3] D. Roder, N. Houssami, G. Farshid, G. Gill, P. Downey, K. Beckmann, P. Iosifidis, L. Grieve, and L. Williamson, "Population screening and intensity of screening are associated with reduced breast cancer mortality: evidence of efficacy of mammography screening in Australia", Breast Cancer Research and Treatment, vol. 108, no. 3, pp. 409-416, 2008.

[4] D. Miglioretti, R. Smith-Bindman, L. Abraham, J. Brenner, E. Aiello, M. Buist, P. Carney, and J. Elmore, "Radiologist characteristics associated with interpretive performance of diagnostic mammography", Journal of the National Cancer Institute, vol. 99, no. 24, pp. 1854-63, 2007.

[5] R. Brem, "Clinical versus research approach to breast cancer detection with CAD: where are we now? American Journal of Roentology, vol. 188, pp. 234-235, 2007.

[6] J. Dean and V. Ilvento, "Improved cancer detection using computer-aided detection with diagnostic and screening mammography: prospective study of 104 cancers", American Journal of Roentology, vol. 187, pp. 20-28, 2006.

[7] R. Birdwell, P. Bandodkar, and D. Ikeda, "Computer-aided detection with screening 
mammography in a university hospital settings", Radiology, vol. 236, pp. 451-457, 2005.

[8] M. Morton, D. Whaley, K. Brandt, and K. Amrami, "Screening mammograms: interpretation with computer-aided detection - prospective evaluation", Radiology, vol. 239, pp. 204-212, 2006.

[9] H. Cheng, X. Shi, R. Min, L. Ju, X. Cai, and H. Du, "Approaches for automated detection and classification of masses in mammograms", Pattern Recognition, vol. 39, no. 4, pp. 464-668, 2006.

[10]H. Cheng, X. Cai, X. Chen, L. Hu, and X. Lou "Computer-aided detection and classification of microcalcifications in Mammograms: a survey", Pattern Recognition, vol. 36, pp. 2967-91, 2003.

[11] S. Halkiotis, T. Botsis, and M. Rangoussi, "Automatic detection of clustered microcalcifications in digital mammograms using mathematical morphology and neural networks", Signal Processing, vol. 87, pp. 1559-1568, 2007.

[12] P. Delogu, M. Fantacci, P. Kasae, and A. Retico, "Characterization of mammographic masses using a gradient based segmentation algorithm and a neural classifier", Computers in Biology and Medicine, vol. 37, pp. 1479-1491, 2007.

[13]B. Verma, "Novel network architecture and learning algorithm for the classification of mass abnormalities in digitized mammograms", Artificial Intelligence in Medicine, vol. 42, no. 1, pp. 67-79, 2008.

[14]B. Verma and R. Panchal, "Neural networks for the classification of benign and malignant patterns in digital mammograms", Advances in Applied Artificial Intelligence, USA, Book Editor: John Fulcher, 2006.

[15] P. Zhang, B. Verma, and K. Kumar, "A neural-genetic algorithm for feature selection and breast abnormality classification in digital mammography", Proc. IEEE-IJCNN, vol.3, pp. 2303-09, 2004.

[16]P. Zhang, B. Verma, and K. Kumar, Neural Vs. Statistical Classifier in Conjunction with Genetic Algorithm Feature Selection in Digital Mammography, Pattern Recognition Letters, 26(7), pp. 909-919, 2005.

[17] B. Verma and J. Zakos, "A computer-aided diagnosis system for digital mammograms based on fuzzy-neural and feature extraction techniques", IEEE Transactions on Information Technology in Biomedicine, vol. 5, pp. 46-54, 2001.

[18] M. Bicegoa and M. Figueiredo, Soft clustering using weighted one-class support vector machines, Pattern Recognition, vol. 42, pp. 27 - 32, 2009.

[19]H. Georgiou, M. Mavrofarakis, N. Dimitropoulos, D. Cavouras, and S. Theodoridis, "Multi-scaled morphological features for the characterization of mammographic masses using statistical classification schemes", Artificial Intelligence in Medicine, vol. 41, pp. 39-55, 2007.

[20] R. Brem, J. Hoffmeister, G. Zisman, M. Simio, and S. Rogers, "A computer aided detection system for the evaluation of breast cancer by mammographic appearance and lesion size", American Journal of Roentology, vol. 184, pp. 893-896, 2004.
[21] Y. Bi, J. Guan and D. Bell, "The combination of multiple classifiers using an evidential reasoning approach", Artificial Intelligence, vol. 172, pp. 1731-1751, 2008.

[22] Y. Chen and Y. Zhao, "A novel ensemble of classifiers for microarray data classification, Applied Soft Computing", vol. 8, pp. 1664-1669, 2008.

[23] G. Tourassi, B. Haarawood, S. Singh, J. Lo, and C. Floyd, "Evaluation of information-theoretic similarity measures for content based retrieval and detection of masses in mammograms", Medical Physics, vol. 34, pp. 140-150, 2007.

[24] M. Massotti, "A ranklet-based image representation for mass classification in digital mammograms", Medical Physics, vol. 33, no. 10, pp. 3951-3961, 2006.

[25] R. Acharya, U. Ng, Y. Chang, J. Yang, and G. Kaw, "Computer based identification of breast cancer using digitized mammograms", Journal of Medical Systems, 2008, doi:10.1007/s10916-008-9156-6.

[26] M. Heath, K. Bowyer, D. Kopans, R. Moore, and P. Kegelmeyer, "The Digital Database for Screening Mammography", IWDM-2000, Medical Physics Publishing, 2001. 